\title{
A STUDY ON THE MOTION CHANGE UNDER LOADED CONDITION INDUCED BY VIBRATION STIMULATION ON BICEPS BRACHII
}

\author{
Koki Honda $^{1}$ and Kazuo Kiguchi ${ }^{2}$ \\ ${ }^{1,2}$ Department of Mechanical Engineering, Kyushu University, Fukuoka, Japan
}

\begin{abstract}
To assist not only motor function but also perception ability of elderly and/or handicapped persons, the power-assist robots which have perception-assist function have been developed. These robots can automatically modify the user's motion when the robot detects inappropriate user's motion or a possibility of accident such as collision between the user and obstacles. For this motion modification in perception-assist, some actuators of power-assist robot are used. On the other hand, since some elderly persons, handicapped persons or some workers need not use power-assist function but perception-assist function only, another new concept perception-assist method was investigated in our previous study. In this perception-assist method, only vibrators are used for generating motion change with kinesthetic illusion to assist perception-ability only. In this study, since the perception-assist is often used during tasks under a loaded condition, the features of motion change under the loaded condition are investigated.
\end{abstract}

\section{KEYWORDS}

Vibration Stimulation, Perception-Assist, Motion Change, Elbow Joint Motion

\section{INTRODUCTION}

Since not only motor function but also perception ability to surrounding environment are deteriorated in some elderly and/or handicapped persons, some power-assist exoskeleton robots which have perception-assist function have been proposed [1], [2]. The power-assist robot with perception-assist function keeps monitoring the interaction between the user and the environment by using its sensors and if the user is making inappropriate motion or dangerous motion, the robot modifies his/her motion automatically by generating additional external force with some actuators.

On the other hand, there are also some elderly persons, handicapped persons and workers whose motor function is not so deteriorated but only perception ability has problem. To assist such persons, another new concept perception-assist method has been proposed in our previous study [3], [4]. In this perception-assist method, a vibrator is used to generate motion change with kinesthetic illusion and it has a possibility to realize an effective perception-assist device.

When vibration stimulation is added to the tendon of antagonist muscle around a human joint, a person feel as if their antagonist is elongated and feel as if their joint is rotating. This Dhinaharan Nagamalai et al. (Eds) : CoSIT, SIGL, AIAPP, CYBI, CRIS, SEC, DMA - 2017 
phenomenon is called as "kinaesthetic illusion" and it is discovered by Goodwin in 1972 [5]. This kinaesthetic illusion has been studied in the field of neurophysiology. The cause of this illusion is that the receptors in a muscle, called as muscle spindle, is stimulated by vibration and it generate electric signals, called as Ia afferents, to brain. By receiving this Ia afferents, the brain makes misinterpretation that the vibrated muscle is elongated and the body parts which is connected to vibrated muscle are moving despite the body parts are not moving actually [6], [7].

We found that the motion change can be generated by using this misinterpretation generated by vibration stimulation in previous studies [3], [4]. In those studies, vibration stimulation is added to biceps brachii and triceps brachii during elbow joint flexion/extension motion and owing to the gap between actual elbow joint angular velocity and subject's elbow joint feeling, motion change was generated.

In this study, vibration stimulation is added to biceps brachii during elbow extension motion under some loaded conditions. Since many daily tasks are conducted under a loaded condition, the features of motion change under the loaded condition induced by vibration stimulation must be investigated. The experimental results show that the motion change can be generated under the loaded condition by vibration stimulation and the changing rates of motion change are investigated.

\section{METHODS}

\subsection{Experimental devices}

In this study, vibration stimulation is added to biceps brachii of subject's right elbow. Subjects are seated on a chair (see Figs. 1 and 2). Subject's both arms are fixed to the frame of goniometer (rotary encoder: RE12D-300-201). The angle range of elbow extension motion is 0 (deg)-45 (deg). Elbow angle and angular velocity are recorded by workstation. According to previous study [3], 70-100 Hz vibration stimulation can generate kinaesthetic illusion and motion change. The vibrator used in this study (Fig.1) can also generate 70-100 (Hz) vibration stimulation and its amplitude is $1.0(\mathrm{~mm})$.

\subsection{Procedure of the experiments}

In this experiment, the effect of loaded condition to motion change caused by vibration stimulation is investigated. To make the amount of motion change with kinaesthetic illusion clear, subjects are assigned to make the elbow angle of the right "Vibrated arm" correspond to the elbow angle of left "Reference arm". It is assumed that a gap between the elbow angle of Vibrated arm and Reference arm is generated when an illusion is generated with vibration

stimulation. Procedure of the experiment is shown below. There are a practice part and an experiment part.

A) Practice part

1. Practices are conducted to position their elbow angle of both arm 20 (deg) without watching.

2. Other practices are conducted to generate extension motion with both elbow angles' angular velocity $3(\mathrm{deg} / \mathrm{s})$. 
B) Experiment part

1. Subject's elbow angle is adjusted to 0 (deg).

2. The encoders recording are started and subject's both elbow angles are adjusted to 45 (deg).

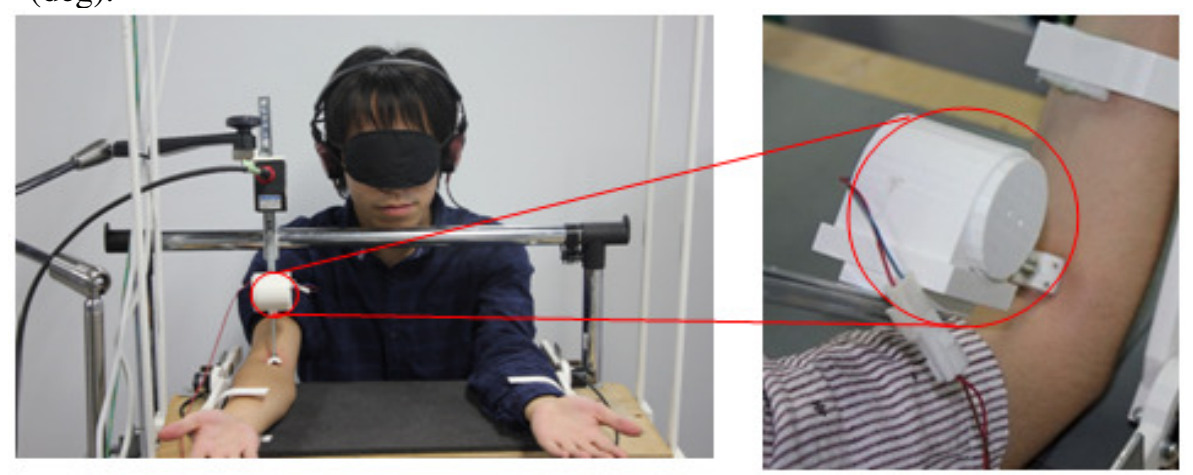

Figure 1. Experimental device and vibrator

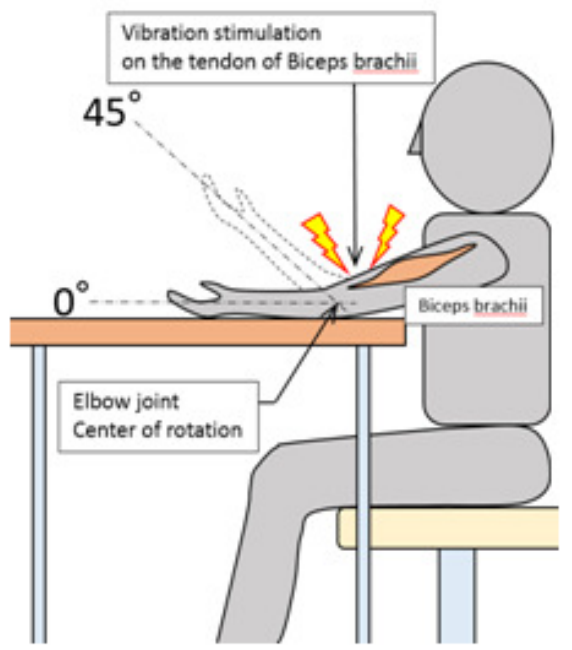

Figure 2. Experimental conditions

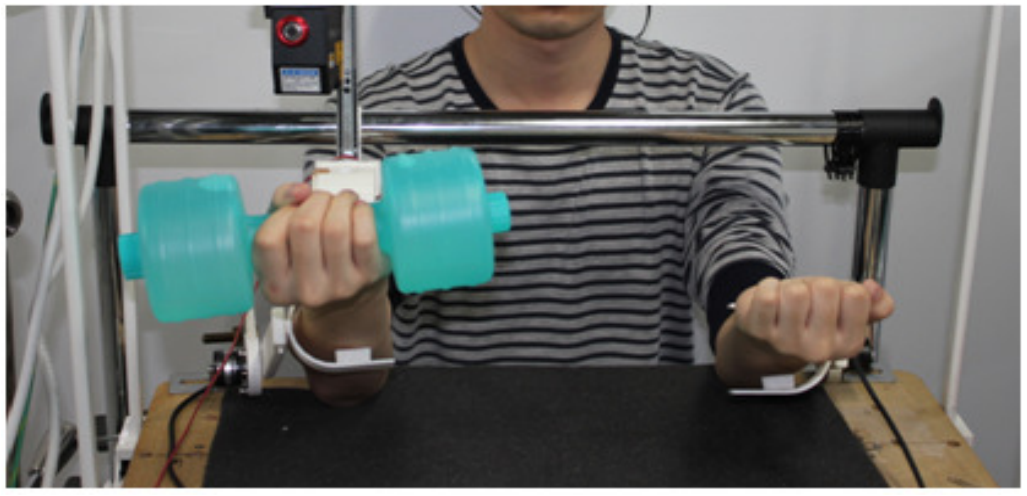

Figure 3. Loaded condition during motion change 
3. Experimenter gives a weight to the subject and the subject hold the weight by his/her hand (see Fig. 3). After that, vibration stimulation is started and then extension motion with $3(\mathrm{deg} / \mathrm{s})$ of both arms are started 10 seconds later.

4. When the reference arm comes up to 45(deg), encoders are turned off and vibration stimulation is ended.

5. Once trial is finished, a weight is changed by the experimenter and the next procedure is restarted.

Subjects are shown as bellow (Table 1). We have 3 patterns of loaded conditions; 1000 (g), 500 $(\mathrm{g})$, and $0(\mathrm{~g})$. Subject's eyes are closed and subjects hear white noise during the experiment.

Table 1. Subjects

\begin{tabular}{|c|c|c|c|c|}
\hline & Sex & Age & Weight (kg) & Height (cm) \\
\hline \hline Subject1 & Man & 23 & 57 & 166 \\
\hline Subject2 & Man & 25 & 57 & 156 \\
\hline Subject3 & Man & 23 & 62 & 178 \\
\hline
\end{tabular}

\section{RESULTS}

Trials are conducted 3 times on each loaded conditions with each subject. From the records of subject's elbow joint angle during the experiments, the changing rate of the gap between the Reference arm and the Vibrated arm is calculated (see Fig. 4). In almost all subjects, the data of the elbow angles of both arms during $13 \mathrm{~s}$ to $18 \mathrm{~s}$, after vibration stimulation start, are used in this calculation. The average of the changing rates in 3 trials and elbow angle of the Vibrated arm are shown in Table 2.

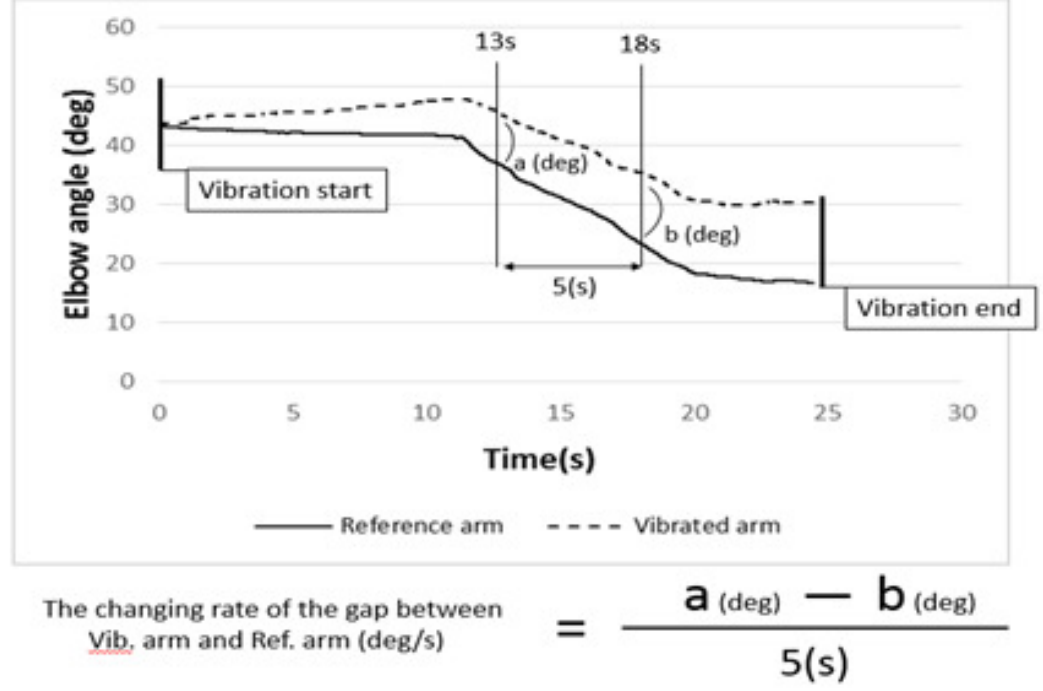

Figure 4. Calculation Method 
Table 2 Results of experiments

\begin{tabular}{|c|c|c|c|c|}
\hline \multicolumn{2}{|c|}{} & $\mathbf{0}(\mathbf{g})$ & $\mathbf{5 0 0}(\mathbf{g})$ & $\mathbf{1 0 0 0}(\mathbf{g})$ \\
\hline \hline \multirow{3}{*}{ Subject1 } & Changing rate(deg/s) & 0.8 & 1.2 & 1.5 \\
\cline { 2 - 5 } & Angle (deg) & 45 & 40 & 38 \\
\hline \multirow{3}{*}{ Subject2 } & Changing rate(deg/s) & 1.6 & 1.2 & 1.4 \\
\cline { 2 - 5 } & Angle (deg) & 42 & 36 & 44 \\
\hline \multirow{2}{*}{ Subject3 } & Changing rate(deg/s) & 2.4 & 3.1 & 1.6 \\
\cline { 2 - 5 } & Angle (deg) & 44 & 46 & 48 \\
\hline
\end{tabular}

\section{DISCUSSION}

In all subjects, the gap between the Vibrated arm's elbow angle and the Reference arm's elbow angle was generated. These experimental results suggest that motion change can be generated under loaded condition by vibration stimulation.

The correlation between the increasing of load and the increasing or decreasing of changing rate was not seen clearly in these experiments. There is a possibility that the kinaesthetic illusion is enhanced as the load increases since the muscle spindles are more strongly elongated by load. On the other hand, as the angle of the elbow joint decreases, however, the moment due to the load applied to the elbow joint increases because of the gravity effect. Consequently, there is a possibility that the gap between the Vibrated arm's elbow angle and the Reference arm's elbow angle was decreased by the effect of the moment.

The results suggests that there is a possibility that the correlation between the load increase and the increase/decrease of the changing rate can be obtained more clearly if the constant torque is added to the Vibrated arm's elbow joint during its extension motion.

\section{ACKNOWLEDGEMENTS}

This work was partially supported by Japan Society of Promotion of Science (JSPS) Grant-in-Aid for Scientific Research (B) 16H04305.

\section{REFERENCES}

[1] K. Kiguchi, M. Liyanage, and Y. Kose (2009) "Perception Assist with an Active Stereo Camera for an Upper-Limb Power-Assist Exoskeleton”, Int. Journal of Robotics and Mechatronics, vol. 21, no. 5, pp.614-620.

[2] K. Kiguchi and Y. Hayashi (2011) "A Lower-Limb Power-Assist Robot with Perception-Assist", Proc. of IEEE Int. Conf. on Rehabilitation Robotics, pp.731-736. 
[3] Koki Honda, Kazuo Kiguchi (2016) "A Fundamental Study on the Effect of Vibration Stimulation for Motion Modification in Perception-Assist", Proc. of IEEE Int. Conf. on Systems, Man and Cybernetics.

[4] Koki Honda, Kazuo Kiguchi (2016) " A Fundamental Study on the Effect of Vibration Stimulation on Triceps Brachii during Elbow Flexion motion for Perception-Assist”, Proc. of Int. Symp. on MicroNanomechatronics and Human Science.

[5] G. M. Goodwin, D. I. McCloskey, P. B. C. Matthews (1972) "The contribution of muscle afferents to kinaesthesia shown by vibration induced illusions of movement and by the effects of paralysing joint afferents.” Brain, Vol.95, No.4, pp.705-748.

[6] E. Naito, H.H. Ehrsson, S. Geyer, K. Zilles, and P.E. Roland (1999) "Illusory arm movements activate cortical motor areas: a positron emission tomography study", The Journal of neuroscience, vol.19, no.14, pp.6134-6144.

[7] J.P. Roll and J.P. Vedel (1982) "Kinaesthetic role of muscle afferents in man, studied by tendon vibration and microneurography”, Experimental Brain Research, vol.47, no.2, pp.177-190.

\section{AUTHORS}

Koki Honda received B. Eng. Degree in mechanical engineering from Kyushu University, Fukuoka, Japan, in 2015, He is currently master course student of Kyushu University, Fukuoka, Japan.

Kazuo Kiguchi received the B. Eng. degree in mechanical engineering from Niigata University, Niigata, Japan, in 1986, the M. A Sc. degree in mechanical engineering from the University of Ottawa, Ottawa, Canada, in 1993, and the Dr. Eng. degree from Nagoya University, Nagoya, Japan, in 1997. He is currently a professor in the Dept. of Mechanical Engineering, Faculty of Engineering, Kyushu University, Japan.
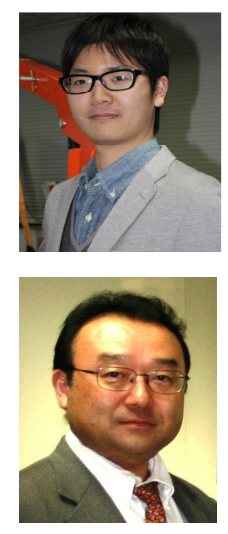